\title{
Cardiac arrhythmias and rhabdomyolysis in Bartter-Gitelman patients
}

\author{
Cinzia Cortesi • Sebastiano A. G. Lava • Alberto Bettinelli • Fabiana Tammaro • \\ Olivier Giannini • Maria Caiata-Zufferey • Mario G. Bianchetti
}

Received: 8 April 2010/Revised: 11 May 2010/Accepted: 17 May 2010/Published online: 13 June 2010 (C) IPNA 2010

\begin{abstract}
Recent data demonstrate that patients affected with hypokalemic salt-losing tubulopathies are prone to acute cardiac arrhythmias and rhabdomyolysis. The tendency to these potentially fatal complications is especially high if chronic hypokalemia is severe, in patients with diarrhea, vomiting or a prolonged QT interval on standard electrocardiography, in patients on drug management with compounds prolonging the electrocardiographic QT interval (including antiarrhythmic agents, some antihistamines, macrolides, antifungals, psychotropics, $\beta_{2}$-adrenergic agonists or cisapride), following acute alcohol abuse and during exercise. Cardiac arrhythmias and rhabdomyolysis occur with sufficient
\end{abstract}

C. Cortesi - S. A. G. Lava $\cdot$ M. G. Bianchetti

Department of Pediatrics, Mendrisio and Bellinzona Hospitals,

University of Bern,

Bern, Switzerland

\author{
A. Bettinelli $\cdot$ F. Tammaro \\ Department of Pediatrics, San Leopoldo Mandic Hospital, \\ Merate, Italy \\ O. Giannini \\ Nephrology and Internal Medicine Services, \\ Ospedale Regionale Beata Vergine, \\ Mendrisio, Switzerland \\ M. Caiata-Zufferey \\ Institute of Communication and Health, \\ Università della Svizzera Italiana, \\ Lugano, Switzerland \\ M. G. Bianchetti $(\bowtie)$ \\ San Giovanni Hospital, \\ 6500 Bellinzona, Switzerland \\ e-mail: mario.bianchetti@pediatrician.ch
}

frequency in hypokalemic salt-losing tubulopathies to merit wider awareness of their presence and the preparation of specific prevention and management recommendations.

Keywords Bartter syndrome - Cardiac arrhythmia . Gitelman syndrome $\cdot$ Hypokalemia $\cdot$ Hypomagnesemia . Rhabdomyolysis $\cdot$ Sudden cardiac death

Hypokalemic salt-losing tubulopathies, such as Bartter and Gitelman syndromes, are a group of closely related tubular disorders, first described in 1962, which are transmitted as autosomal recessive traits. All BartterGitelman patients share several characteristics, including hypokalemia (with or without concurrent hypomagnesemia), metabolic alkalosis, normal or low blood pressure, and activated renin-angiotensin-aldosterone and prostaglandin-kinin systems [1-3].

Thirteen years ago, a boy with unclassified BartterGitelman syndrome in our care died suddenly [4]. Since potassium depletion, with or without concurrent magnesium depletion, imparts an increased risk for developing cardiac arrhythmias culminating in syncope or even sudden death (this link is very well documented for patients managed with thiazide diuretics), we attributed the death to hypokalemia [5-9].

A prolonged QT interval on standard electrocardiography $[10,11]$ imparts an increased risk for developing a ventricular arrhythmia culminating in syncope or sudden death (Fig. 1). In agreement with this finding, we have observed during years of clinical practice that the QT-interval is often prolonged in patients with Gitelman syndrome, which is the most frequent variant of these syndromes, leading us to speculate that these patients are of an increased risk of developing dangerous cardiac arrhythmias [12]. However, continuous ambulatory electrocardiography and 
exercise testing failed to detect significant abnormalities of cardiac rhythm in our Gitelman patients [13]. Nonetheless, since both hypokalemia and hypomagnesemia were rather mild in these patients, we presumed [14] that dangerous cardiac arrhythmias may occur in patients with severe hypokalemia (and hypomagnesemia), as indicated by the case of a newborn with profound hypokalemia in the context

V2

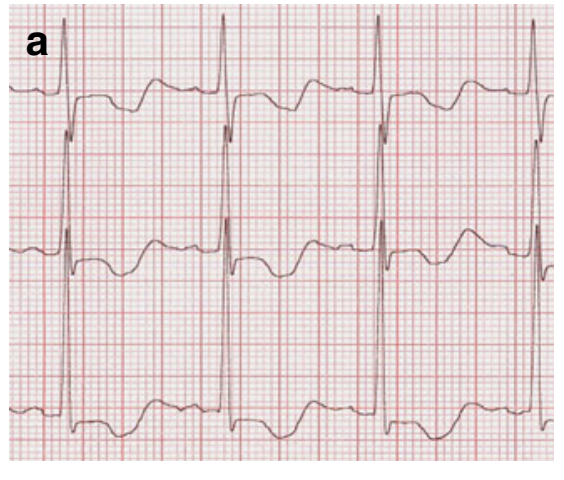

Plasma

Potassium

$1.4 \mathrm{mmol} / \mathrm{L}$

\section{V4}

V2b

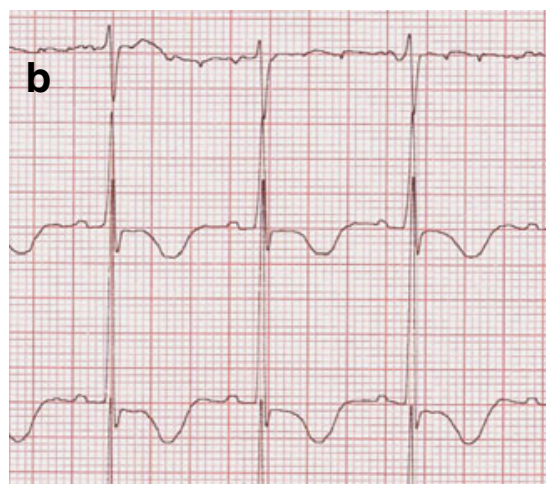

Plasma

Potassium

$1.8 \mathrm{mmol} / \mathrm{L}$

V4

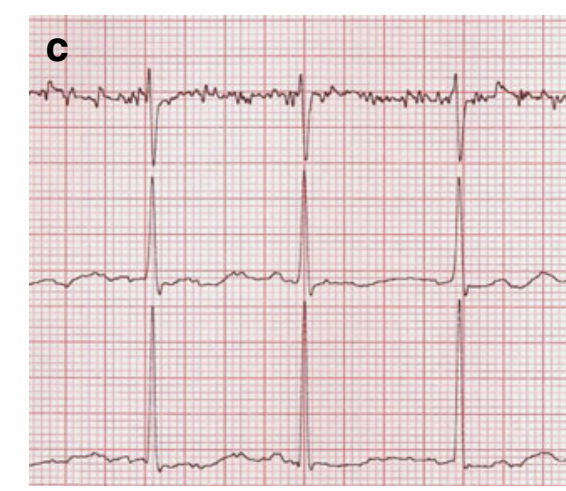

\section{Plasma}

Potassium

\section{$2.0 \mathrm{mmol} / \mathrm{L}$}

Fig. 1 Electrocardiographic changes induced by hypokalemia are a flat or even inverted T wave (a), a depressed ST segment (b), and the presence of a $U$ wave (c). Other findings, especially in the presence of coexistent hypomagnesemia, include a prolonged QT interval, ventricular extrasystoles, ventricular tachycardia, torsades de pointes, and ventricular fibrillation. The figure depicts the electrocardiographic signals in a female patient with the presumed diagnosis of Bartter syndrome admitted with a potassium level of $1.4 \mathrm{mmol} / \mathrm{L}$. The figure illustrates electrocardiography at presentation and following an initial correction of hypokalemia of the antenatal form of Bartter syndrome, who developed severe cardiac arrhythmias and cardiac arrest [15].

Considering the persisting uncertainty related to the possible occurrence of cardiac arrhythmias culminating in syncope or sudden death in the context of Bartter-Gitelman syndromes, we conducted a survey/inquiry among physicians caring for Bartter-Gitelman children, which disclosed four patients with lethal and three with severe non-lethal cardiac arrhythmias [16]. Six recent case reports support the assumption that hypokalemia may cause dangerous cardiac arrhythmias in Bartter-Gitelman patients [17-21].

In addition to its potential to cause cardiac arrhythmias, severe hypokalemia may effect dissolution of the peripheral striated muscle fibers with leakage of the intracellular constituents, a distinctive condition known as rhabdomyolysis $[22,23]$. The immediate consequences of rhabdomyolysis include hyperkalemia (and hyperphosphatemia) resulting from the release of potassium and phosphorous from damaged muscle, which in turn may cause cardiac arrhythmias, and acute kidney injury. In fact, two recent reports indicate that Bartter-Gitelman patients occasionally do develop rhabdomyolysis [24, 25].

Based on the afore-mentioned data, we offer some takehome messages relevant to the prevention of hazardous cardiac arrhythmias and rhabdomyolysis in patients affected with hypokalemic salt-losing tubulopathies. The value of our advice should be confirmed in long-term observational studies.

1) Severe chronic hypokalemia $(\leq 2.5 \mathrm{mmol} / \mathrm{L})$ is hazardous [5-7]. Consequently, in addition to potassium salts and cyclooxygenase inhibitors $[1,2]$, potassiumsparing diuretics and blockers of the renin-angiotensin-aldosterone system deserve consideration in patients with refractory hypokalemia or with gastrointestinal side effects secondary to potassium supplementation or medication with nonselective anti-inflammatory drugs. However, very limited clinical experience has been reported to date with blockers of the renin-angiotensinaldosterone system in these patients [26, 27].

2) In Bartter-Gitelman patients, acute diarrhea or vomiting [16], even if mild, may cause very severe hypokalemia $(\leq 2.0 \mathrm{mmol} / \mathrm{L})$. In this setting, early referral and prompt electrolyte and fluid repair are of paramount importance, since the repair of hypokalemia with oral potassium supplementation, potassiumsparing diuretics, cyclooxygenase inhibitors, and blockers of the renin-angiotensin-aldosterone system often meets with limited success. In fact, hypokalemia is largely secondary to extracellular fluid volume depletion, a potent stimulus for the renin-angiotensin-aldosterone system [28], suggesting that addressing directly extracellular fluid volume depletion might 
be a further, hitherto rather neglected treatment option for hypokalemia [3]. It is therefore tempting to advise a more liberal fluid and salt intake in order to prevent -at least in part-the tendency toward severe, chronic hypokalemia. Conversely, in patients with acute diarrhea and vomiting, parenteral rehydration with isotonic saline may be as important as direct potassium replacement to treat very severe hypokalemia. One further critical therapeutic principle concerns the intravenous administration of potassium in glucose-free solutions, since this sugar may cause a further decrease in potassium level, precipitating arrhythmias and rhabdomyolysis [7].

3) A prolonged QT interval on standard electrocardiography imparts an increased risk for the development of cardiac arrhythmias, culminating in syncope or sudden death that reflects both acquired (including potassium levels) and congenital factors $[10,11]$. Considering that a prolonged QT interval has been associated with Bartter-Gitelaman syndromes whatever the potassium levels, the QT interval has to be systematically measured in these patients. In those patients with a prolonged QT interval, potassium levels have to be more closely $(\geq 3.0 \mathrm{mmol} / \mathrm{L})$ controlled.

In Bartter patients with a prolonged QT interval (Table 1), as in patients with hypertrophic cardiomyopathy, coronary artery anomalies, Marfan syndrome, aortic stenosis, and Wolff-Parkinson-White syndrome, exercise or participation in recreational and, especially, competitive sports is associated with some risk of sudden cardiac death $[10,11]$. In these patients, a history of syncope is a further powerful predictor of life-threatening cardiac events $[10,11]$. As a consequence, Bartter-Gitelman patients with a prolonged QT interval should avoid getting involved in competitive sports. This recommendation is supported by the results of a study that demonstrated an impaired capacity to adapt left ventricular function in response to exercise in BartterGitelman patients [29]. Considering that the recommendation

Table 1 Recommended values for the corrected QT interval (ms). Reprinted from [10] with permission from Elsevier. The corrected QT interval may be calculated from the QT and the RR intervals (both in $\mathrm{ms}$ ) using the Bazett's equation: $\frac{\mathrm{QT}}{\sqrt{\mathrm{RR}}}$

\begin{tabular}{|c|c|c|c|}
\hline \multirow[t]{2}{*}{ QT interval (ms) } & \multicolumn{3}{|l|}{ Age } \\
\hline & $1-15$ years & Adult Male & Adult Female \\
\hline \multicolumn{4}{|l|}{ Rating } \\
\hline Normal & $<440$ & $<430$ & $<450$ \\
\hline Borderline & $440-460$ & $430-450$ & $450-470$ \\
\hline Prolonged & $>460$ & $>450$ & $>470$ \\
\hline
\end{tabular}

is often hard on adolescents and adults involved in competitive sport activities, we usually seek the advice of a cardiologist.

Finally, in Bartter patients, further promoters of cardiac arrhythmias or rhabdomyolysis deserve consideration.

1) In patients with either a congenital or acquired long QT interval, acute alcohol abuse and a number of compounds (including antiarrhythmic agents, some antihistamines, macrolides, antifungals, psychotropics, $\beta_{2}$ adrenergic agonists or cisapride) may cause a further QT prolongation and, therefore, cardiac arrhythmias $[10,11]$.

2) Conversely, alcohol abuse and both illicit (most frequently cocaine or ecstasy) and prescribed drugs (most frequently statins and antipsychotic drugs) are potentially myotoxic. The spectrum of severity is broad and varies from an asymptomatic increase in blood creatine kinase level to acute rhabdomyolysis. Hence, we assume (but can not prove) that these factors may enhance the tendency towards rhabdomyolysis in Bartter syndrome [22, 23]. Educating Bartter patients, their family members and their family physicians on alcohol abuse and illicit or prescribed drugs that may prolong the QT interval or cause myotoxicity is of tremendous importance.

In conclusion, improving the outcome of rare diseases is of the utmost concern. Cardiac arrhythmias and rhabdomyolysis occur, in our opinion, with sufficient frequency in Bartter-Gitelman patients to merit a wider awareness of their presence and the preparation of specific prevention and management recommendations.

\section{References}

1. Bettinelli A, Vezzoli G, Colussi G, Bianchetti MG, Sereni F, Casari G (1998) Genotype-phenotype correlations in normotensive patients with primary renal tubular hypokalemic metabolic alkalosis. J Nephrol 11:61-69

2. Zelikovic I (2003) Hypokalemic salt-losing tubulopathies: an evolving story. Nephrol Dial Transplant 18:1696-1700

3. Knoers NV, Levtchenko EN (2008) Gitelman syndrome. Orphanet J Rare Dis 3:22

4. Zanolari Calderari M, Vigier RO, Bettinelli A, Bianchetti MG (2002) Electrocardiographic QT prolongation and sudden death in renal hypokalemic alkalosis. Nephron 91:762-763

5. Anonymous (1980) Severe hypokalaemia. Lancet 315:520-521

6. Weiner ID, Wingo CS (1997) Hypokalemia-consequences, causes, and correction. J Am Soc Nephrol 8:1179-1188

7. Gennari FJ (2002) Disorders of potassium homeostasis. Hypokalemia and hyperkalemia. Crit Care Clin 18:273-288

8. Bigger JT Jr (1994) Diuretic therapy, hypertension, and cardiac arrest. N Engl J Med 330:1899-1900

9. Cohen HW, Madhavan S, Alderman MH (2001) High and low serum potassium associated with cardiovascular events in diuretictreated patients. J Hypertens 19:1315-1323 
10. Goldenberg I, Moss AJ (2008) Long QT syndrome. J Am Coll Cardiol 51:2291-2300

11. Zareba W, Cygankiewicz I (2008) Long QT syndrome and short QT syndrome. Prog Cardiovasc Dis 51:264-278

12. Bettinelli A, Tosetto C, Colussi G, Tommasini G, Edefonti A, Bianchetti MG (2002) Electrocardiogram with prolonged QT interval in Gitelman disease. Kidney Int 62:580-584

13. Foglia PE, Bettinelli A, Tosetto C, Cortesi C, Crosazzo L, Edefonti A, Bianchetti MG (2004) Cardiac work up in primary renal hypokalaemia-hypomagnesaemia (Gitelman syndrome). Nephrol Dial Transplant 19:1398-1402

14. Bianchetti MG, Cortesi C, Foglia PE, Edefonti A, Bettinelli A (2003) Myocardial function in Bartter's and Gitelman's syndromes. Kidney Int 64:367

15. Haas NA, Nossal R, Schneider CH, Lewin MA, Ocker V, Holder M, Uhlemann F (2003) Successful management of an extreme example of neonatal hyperprostaglandin-E syndrome (Bartter's syndrome) with the new cyclooxygenase-2 inhibitor rofecoxib. Pediatr Crit Care Med 4:249-251

16. Cortesi C, Bettinelli A, Emma F, Fischbach M, Bertolani P, Bianchetti MG (2005) Severe syncope and sudden death in children with inborn salt-losing hypokalaemic tubulopathies. Nephrol Dial Transplant 20:1981-1983

17. Nakane E, Kono T, Sasaki Y, Tokaji Y, Ito T, Sohmiya K, Sakai Y, Suwa M, Tanaka T, Nisimura H, Kitaura Y (2004) Gitelman's syndrome with exercise-induced ventricular tachycardia. Circ J 68:509-511

18. Malafronte C, Borsa N, Tedeschi S, Syren ML, Stucchi S, Bianchetti MG, Achilli F, Bettinelli A (2004) Cardiac arrhythmias due to severe hypokalemia in a patient with classic Bartter disease. Pediatr Nephrol 19:1413-1415
19. Pachulski RT, Lopez F, Sharaf R (2005) Gitelman's not-so-benign syndrome. N Engl J Med 353:850-851

20. Darbar D, Sile S, Fish FA, George AL Jr (2005) Congenital long QT syndrome aggravated by salt-wasting nephropathy. Heart Rhythm 2:304-306

21. Scognamiglio R, Negut C, Calò LA (2007) Aborted sudden cardiac death in two patients with Bartter's/Gitelman's syndromes. Clin Nephrol 67:193-197

22. Elsayed EF, Reilly RF (2010) Rhabdomyolysis: a review, with emphasis on the pediatric population. Pediatr Nephrol 25:7-18

23. Melli G, Chaudhry V, Cornblath DR (2005) Rhabdomyolysis: an evaluation of 475 hospitalized patients. Medicine (Baltimore) 84:377-385

24. von Vigier RO, Ortisi MT, La Manna A, Bianchetti MG, Bettinelli A (2010) Hypokalemic rhabdomyolysis in congenital renal tubular disorders - a case-series and a systematic review. Pediatr Nephrol $25: 861-866$

25. Kumagai H, Matsumoto S, Nozu K (2010) Hypokalemic rhabdomyolysis in a child with Gitelman's syndrome. Pediatr Nephrol 25:953-955

26. Aurell M, Rudin A (1981) Effects of captopril in Bartter's syndrome. N Engl J Med 304:1609

27. Hené RJ, Koomans HA, Boer P, Dorhout Mees EJ (1982) Longterm treatment of Bartter's syndrome with captopril. Br Med J (Clin Res Ed) 285:695

28. Nicholls MG, Robertson JI (2000) The renin-angiotensin system in the year 2000. J Hum Hypertens 14:649-666

29. Scognamiglio R, Calò LA, Negut $C$, Coccato $M$, Mormino $P$, Pessina AC (2008) Myocardial perfusion defects in Bartter and Gitelman syndromes. Eur J Clin Invest 38:888-895 